\title{
Tumor-induced inflammation alters neutrophil phenotype and disease progression
}

\author{
Charaf Benarafa(1)
}

\begin{abstract}
Neutrophils are essential to combat infectious agents but contribute to collateral inflammatory damage. Likewise, neutrophils can kill cancer cells and have been shown to promote malignant growth and metastasis through immunosuppressive functions. Two articles in a recent issue of Nature reveal new mechanisms by which tumors induce changes in neutrophil phenotype through production of inflammatory cytokines. Although the two studies report different outcomes on the effects of neutrophils on tumor growth and metastasis, they delineate novel molecular pathways influencing neutrophil phenotype that may provide new approaches to harnessing neutrophil functions in the treatment of cancer.
\end{abstract}

Neutrophils develop in the bone marrow and rapidly respond to danger signals by a prompt mobilization to injured tissues, where they accumulate, amplify inflammatory responses, eliminate pathogens, and sometimes induce local tissue injury. Recent studies indicate that neutrophils can acquire phenotypic changes with markedly altered functions and increased survival [1]. In cancer patients and tumor-bearing mice, a major question has been the elusive origin and functional characterization of tumor-associated neutrophils and granulocytic myeloidderived suppressor cells (G-MDSCs), which can be considered a subset of neutrophils with immunosuppressive activity on T cells. In two recent studies published in Nature [2, 3], tumor-induced mechanisms were shown to alter neutrophil numbers and function, highlighting the plasticity of the neutrophil and its importance in controlling growth and invasiveness of cancer cells.

Correspondence: charaf.benarafa@tki.unibe.ch

Theodor Kocher Institute, University of Bern, Freiestrasse 1, CH-3012 Bern, Switzerland 
(IL)-1 $\beta$ hijacks a previously described homeostatic cascade that promotes granulopoiesis by inducing IL-17 and granulocyte-colony-stimulating factor (G-CSF) [5]. Interestingly, depletion of immunosuppressive neutrophils and $\gamma \delta \mathrm{T}$ cells particularly impaired early metastatic spread but had little effect on the primary tumor growth. The pro-metastatic function of neutrophils was mediated by immunosuppression of CD8 cytotoxic $\mathrm{T}$ cells. Co-depletion of neutrophils and CD8-positive cells reverted the anti-metastatic phenotype associated with neutrophil depletion. It will be interesting to find out whether these findings are reproduced in other models.

The two studies raise several issues about harnessing inflammatory cytokines in cancer therapy. First, repurposing anti-inflammatory drugs and, more specifically, antibodies blocking inflammatory cytokines that act upstream of G-CSF could be envisaged for reducing neutrophil production. Indeed, G-CSF was shown to be necessary and sufficient to alter hematopoiesis in favor of production of immunosuppressive neutrophils in the PyMT model [6]. However, it is also likely that tumor-induced inflammation concurrently promotes the production of neutrophils with anti-tumor activity, such as $\mathrm{Met}^{+}$neutrophils. Therefore, the net effect of such therapies may be variable depending on the tumor type, the tissue, and the host response. Second, concomitant inflammatory diseases and environmental exposures, such as cigarette smoke, may affect breast cancer growth and metastasis through effects on neutrophil phenotypes. Knowledge of molecular pathways of neutrophil functions in cancer remains patchy. For example, claims were made for the effects of inducible nitric oxide synthase for both pro- and anti-tumoral neutrophils in the two highlighted articles. This apparent contradiction may be due to the fact that the same neutrophil subset carries out both functions or that neutrophil subsets need to be better defined depending on the tumor and its microenvironment. Migratory and localization profiles of neutrophils within the primary tumor, endothelial surfaces, and pre-metastatic niches relative to tumor cells and $\mathrm{T}$ cells may tip the balance in different directions. In vivo live cell imaging of such interactions may be particularly revealing. In conclusion, these studies show that tumor-associated inflammation profoundly alters granulopoiesis and simultaneously releases neutrophils with different migratory and anti-tumor properties. The defined pathways highlighted here provide the basis for further studies to define intervention points to target tumor type-specific neutrophil migration and function.

\section{Abbreviations}

G-CSF: Granulocyte-colony-stimulating factor; HGF: Hepatocyte growth factor; IL: Interleukin; PyMT: Polyoma virus middle T.
Acknowledgments

CB is funded by the Swiss National Science Foundation (310030-149790).

Published online: 06 October 2015

References

1. Kruger P, Saffarzadeh M, Weber ANR, Rieber N, Radsak M, von Bernuth H, et al. Neutrophils: between host defence, immune modulation, and tissue injury. PLoS Pathog. 2015;11:e1004651

2. Finisguerra V, Di Conza G, Di Matteo M, Serneels J, Costa S, Thompson AAR, et al. MET is required for the recruitment of anti-tumoural neutrophils. Nature. 2015;522:349-53.

3. Coffelt SB, Kersten K, Doornebal CW, Weiden J, Vrijland K, Hau C-S, et al. IL-17-producing $\gamma \delta T$ cells and neutrophils conspire to promote breast cancer metastasis. Nature. 2015;522:345-8.

4. Templeton AJ, McNamara MG, Seruga B, Vera-Badillo FE, Aneja P, Ocaña A, et al. Prognostic role of neutrophil-to-lymphocyte ratio in solid tumors: a systematic review and meta-analysis. J Natl Cancer Inst. 2014;106:dju124.

5. Stark MA, Huo Y, Burcin TL, Morris MA, Olson TS, Ley K. Phagocytosis of apoptotic neutrophils regulates granulopoiesis via IL-23 and IL-17. Immunity. 2005;22:285-94.

6. Casbon A-J, Reynaud D, Park C, Khuc E, Gan DD, Schepers K, et al. Invasive breast cancer reprograms early myeloid differentiation in the bone marrow to generate immunosuppressive neutrophils. Proc Natl Acad Sci U S A. 2015;112:E566-75. 